\title{
Out of the closet and into the waiting room: improving care of 2 SLGBTQIA+ patients in the emergency department
}

\author{
Maria Berliant $^{1}\left[\right.$ [ $\cdot$ Scott Odorizzi ${ }^{1} \cdot$ Jennifer Leppard ${ }^{1}$
}

Received: 25 March 2021 / Accepted: 22 September 2021 / Published online: 28 October 2021

(c) The Author(s), under exclusive licence to Canadian Association of Emergency Physicians (CAEP)/ Association Canadienne de Médecine d'Urgence (ACMU) 2021

Keywords 2SLGBTQIA $+\cdot$ LGBTQ+ · Queer $\cdot$ Emergency $\cdot$ Transgender $\cdot$ Medical education $\cdot$ Discrimination

\section{Introduction}

Individuals presenting to the emergency department (ED) in times of need and extreme vulnerability do so expecting to receive non-judgmental and culturally sensitive care. However, this is often not the case for the two-spirit, lesbian, gay, bisexual, transgender, queer, intersex, asexual, and other (2SLGBTQIA+) community who have faced significant healthcare-related stressors and disparities. 2SLGBTQIA+ individuals are at the mercy of a system that has historically been discriminatory towards them. In a 2014 Canadian study on ED use by transgender individuals, 50\% of respondents had negative ED experiences causing $21 \%$ of respondents to avoid seeking care in the ED [1]. Additionally, a 2013 study showed that $6 \%$ of resident physicians did not agree that 2SLGBTQIA+ patients deserved the same quality of care as others [2]. These alarming statistics point to serious systemic healthcare issues faced by this community. There are specific areas in which ED professionals can improve care for this vulnerable population (see Table 1).

\section{Pronouns}

A common issue faced by transgender or gender non-binary patients is the use of inappropriate names or gender pronouns. For trans individuals, "deadnaming"-being referred to by a former name not corresponding to gender identitycan be extremely invalidating and harmful [3]. Irrespective

Maria Berliant

mber1067@uottawa.ca

1 Faculty of Medicine, Department of Emergency Medicine, The Ottawa Hospital, University of Ottawa, Ottawa, ON, Canada of the healthcare professional's intentions, it may be perceived as careless or dismissive at best and outwardly discriminatory at worst. Patient documentation may also be affected with inconsistent name and/or pronouns throughout patient records.

\section{Heteronormativity}

Another issue faced by 2SLGBTQIA+ patients is an assumption of cisgender and heterosexuality. This can cause a breakdown in the patient-physician relationship and introduce a high degree of mistrust. This can create difficulty in obtaining an accurate history and ensuring appropriate patient care. A 2018 study on resident attitudes, behaviour, and comfort with caring for lesbian, gay, bisexual, transgender, and queer patients showed that a majority $(63 \%)$ of emergency medicine residents neglected to ask about sexual orientation within their work up for abdominal or genital complaints [2].

There may also be unconscious heteronormativity in history-taking during patient interactions. When discussing sexual health, female-presenting patients may be asked "What form of birth control do you use?". This assumes pregnancy is a possible outcome of their sexual activity. Questions such as, "Are you sexually active?" often do not have a simple yes/no answer for a 2SLGBTQIA+ individual. The heteronormative definition of sexual activity often excludes oral sex, manual sex, and sharing of sex objects, thus resulting in incomplete sexual histories.

The assumption of cisgender identity can also affect gender non-conforming individuals for whom presentations may be missed in the differential diagnosis: for example, chest pain as a complication of chest binding-a common practice to minimize breast tissue appearance and alleviate gender 
Table 1 Definitions

\begin{tabular}{ll}
\hline Term & Meaning \\
\hline $\begin{array}{l}\text { Sex } \\
\text { Gender }\end{array}$ & $\begin{array}{l}\text { Usually assigned at birth based on particular physical characteristics: male, female, or intersex } \\
\text { A personal and social construct of roles, expressions, and behaviours that identify one as a man or a woman in a binary } \\
\text { gender system and include a variation of genders within a non-binary system }\end{array}$ \\
2SLGBTQIA+ & $\begin{array}{l}\text { An acronym that stands for "two spirit, lesbian, gay, bisexual, transgender, queer, intersex, asexual, and other" and is used } \\
\text { to describe both sexual orientations and gender identities that fall outside of heterosexuality and cisgender identity }\end{array}$ \\
$\begin{array}{l}\text { Heterosexuality } \\
\text { Aisgender }\end{array}$ & $\begin{array}{l}\text { Denoting a person whose personal gender identity corresponds to their sex assigned at birth } \\
\text { Transgender }\end{array}$ \\
$\begin{array}{l}\text { Denoting a person whose personal gender identity does not correspond to their sex assigned at birth. Often used as a } \\
\text { blanket term and can encompass gender non-binary individuals }\end{array}$ \\
$\begin{array}{l}\text { Not conforming to the social and personal expectations of a binary gender system. Eg., not identifying as male or female } \\
\text { Theteronormativity } \\
\text { The belief that heterosexuality is the "normal" and "default" sexual orientation and the explicit and implicit manner } \\
\text { in which that belief is expressed- for example the lack of expectation to "come out" or reveal oneself as heterosexual } \\
\text { within society }\end{array}$
\end{tabular}

dysphoria. It can be challenging for 2SLGBTQIA+ patients to correct the assumption of cisgender or heterosexuality due to a fear of discrimination from healthcare professionals after disclosing their sexuality and/or gender identity.

\section{Discrimination}

Discrimination and stigma are significant issues faced by 2SLGBTQIA+ individuals, whether outwardly expressed or internalized by healthcare professionals. In one study, $52 \%$ of gender non-conforming individuals had trans-specific negative experiences which varied from perception of healthcare professional discomfort with their gender/ sexuality to outright denial of medical care during their ED visit [1]. Due to a prior personal or community history of discrimination from the medical community, some 2SLGBTQIA+individuals may also have preconceived concerns about the care they will receive. Whether or not this assumption represents the actual quality of care, professionals must be cognizant of the history of the institution they represent and actively address this disparity.

\section{Medical education}

A lack of understanding of 2SLGBTQIA+ specific healthcare needs from healthcare professionals is another area in need improvement. In the American National Transgender Discrimination Survey, $50 \%$ of trans people have had to educate medical staff on their specific healthcare needs [4]. This is in keeping with the literature indicating that less than $10 \%$ of Canadian medical students felt that they were sufficiently knowledgeable to manage transgender-specific health issues, with only $24 \%$ feeling that they had received enough formal teaching on the issue [5]. A healthcare professional who is not knowledgeable about 2SLGBTQIA+ health issues cannot deliver the highest quality of care to the population.

\section{Screening}

The 2SLGBTQIA+ community is at higher risk of substance use, cancer, mental illness, and suicide [6]. Strikingly, youth within a sexual minority are fourteen times more likely to attempt suicide as compared to their heterosexual counterparts and $45 \%$ of transgender respondents in an Ontario study had attempted suicide [7]. This may be related to social and cultural stigma against 2SLGBTQIA+ individuals and a lack of acceptance within the individual's community [7]. Despite these clear risk factors, it is not common practice to inquire about a patient's gender identity or sexuality when the patient presents to the ED with suicidal ideation. Without this information, physicians may not be able to fully understand the social determinants of a patient's mental health which may lead to suboptimal treatment plans [8].

\section{Steps for change}

Given the challenges faced by 2SLGBTQIA+ people within the ED, there must be clear targets for improvement. These changes must be made on both individual and systemic levels and should address the barriers to safe and informed care outlined above. 


\section{Using appropriate name and pronouns}

Ensuring that professionals are respectful and cognizant of a patient's name and pronouns significantly improves the physician-patient relationship for trans and non-binary patients. A simple question such as "how would you like me to refer to you?" can provide patients with an opportunity to disclose their personal name and pronouns. Once disclosed, names and/or pronouns should be clearly recorded in the patient's chart as part of the registration process, which may decrease the likelihood of harmful misgendering and "deadnaming". Gender options other than male/female should be available to register non-binary patients within any charting system.

\section{Using non-assumptive language}

When sexual history is pertinent to a patient's ED presentation, always approach the question without assuming heterosexuality. Leading questions such as "What form of birth control do you use?" or "Do you have a girlfriend?" can create an unwelcoming environment for 2SLGBTQIA+individuals. Using gender-neutral identifiers such as "partner", "spouse", or "significant other" can be a less heteronormative alternative. As mentioned above, sexual activity may have a broader definition for a 2SLGBTQIA+ individual and history taking in the ED must reflect that possibility. Examples of inclusive questions are "What forms of sexual activity do you engage in?" and "What do you do to prevent sexually transmitted infections?".

\section{Screening and appropriate history taking}

Given the health disparities experienced by 2SLGBTQIA+ individuals, it may be beneficial to broaden screening questions for specific issues. The ED is often the first point of healthcare contact for individuals experiencing suicidal ideation and as mentioned above, 2SLGBTQIA+individuals are at a significantly higher risk of depression, self-harm, and suicidal ideation [7]. While it is incorrect to assume that every 2SLGBTQIA+ person struggles with their mental health, there may be a benefit to screening patients with suicidal ideation for distress regarding their gender or sexuality. This could also assist ED physicians in recommending more appropriate outpatient referrals and recommendations to
2SLGBTQIA+friendly counselling services, a trans health clinic, or support groups.

\section{Visible signs of support}

Another important measure to improve the experience of 2SLGBTQIA+ patients in the ED is to show visible signs of support for the community. Examples such as rainbow and trans flags and stickers, posters featuring 2SLGBTQIA+individuals, gender-neutral bathrooms, and healthcare worker badges with visible pronouns can all be perceived as signs of support. Some departments may even create formal policies surrounding the care of patients from a sexual and/ or gender minority. While these displays do not protect the patient against negative healthcare experiences, they can help alleviate expectations of discrimination and enhance patient comfort in the ED setting.

\section{Education}

Finally, healthcare professionals must receive formal education on 2SLGBTQIA+ health. Patients should not be obligated to educate their healthcare team on 2SLGBTQIA+ health issues - especially when they are in a vulnerable situation. Although healthcare professionals are taught to treat all patients equally, we must recognize implicit bias towards minority populations and actively work towards improving the care we provide. Implementation of a medical school curriculum on 2SLGBTQIA+ health has already been found to improve students' understanding of 2SLGBTQIA+ health disparities [9]. These initiatives should be expanded to residency programs and continued professional development activities for attending physicians for further impact. There is immense value in establishing leadership for 2SLGBTQIA+ issues within both large scale and local medical communities. This leadership can pioneer new initiatives and encourage other healthcare professionals to familiarize themselves with local resources and organizations that support 2SLGBTQIA+individuals.

\section{Conclusion}

Historically, medical practitioners have assumed that their patients are heterosexual cisgender individuals, which creates disparities in the care offered to many sexual and gender minority groups. By taking steps to improve the use of appropriate language, address education deficits, and improve provision of 2 SLGBTQIA+ specific care, the ED 


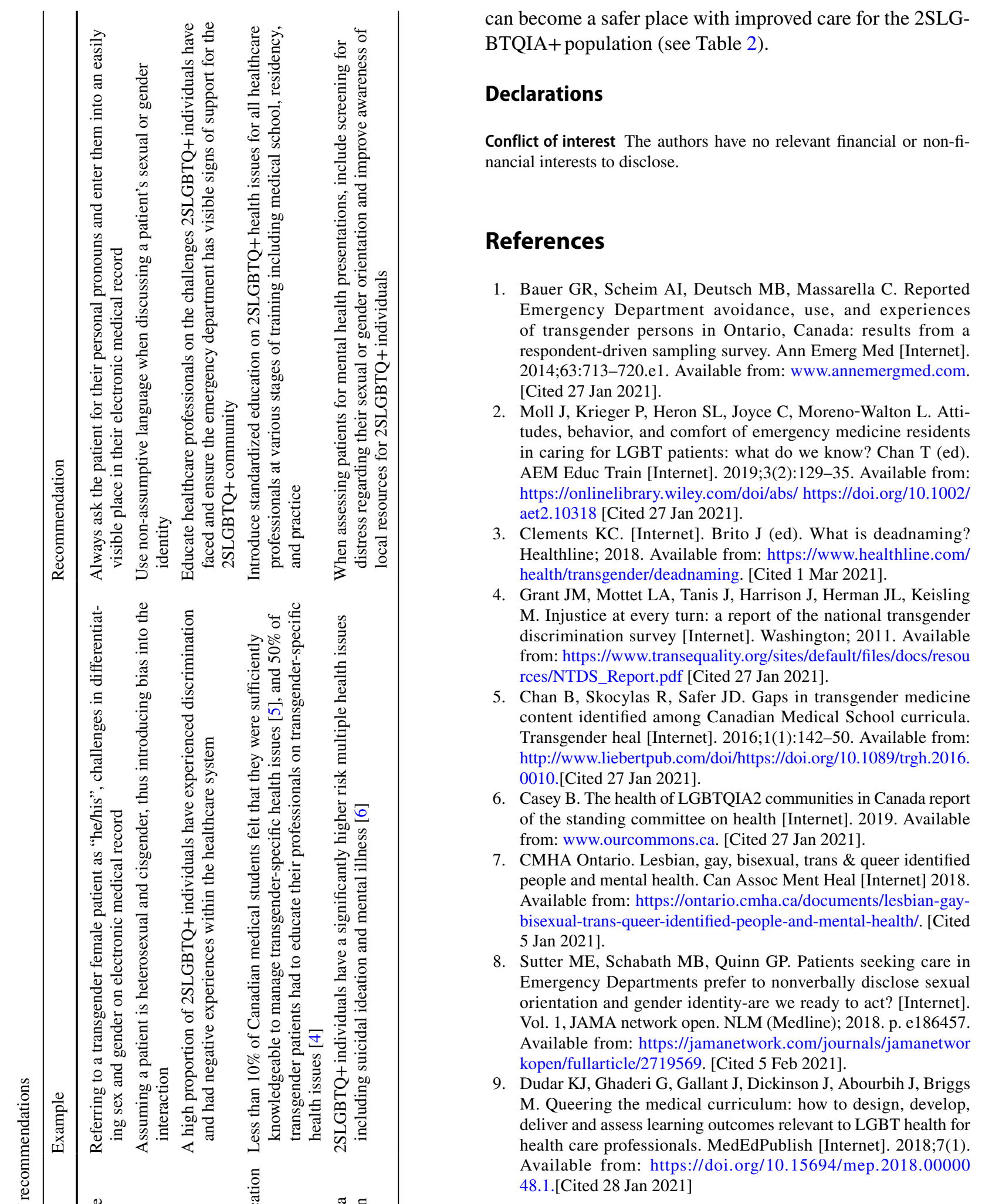

can become a safer place with improved care for the 2SLG(see Table 2).

Conflict of interest The authors have no relevant financial or non-financial interests to disclose.

\section{References}

Bauer GR, Scheim AI, Deutsch MB, Massarella C. Reported Emergency Department avoidance, use, and experiences of transgender persons in Ontario, Canada: results from a 2014;63:713-720.e1. Available from: www.annemergmed.com. 20 Jan 2021] AEM Educ Train [Internet]. 2019;3(2):129-35. Available from: https://onlinelibrary.wiley.com/doi/abs/ https://doi.org/10.1002/

Grant JM, Mottet LA, Tanis J, Harrison J, Herman JL, Keisling M. Injustice at every turn: a report of the national transgender discrimination survey [Internet]. Washington; 2011. Available Transgender heal [Internet]. 2016;1(1):142-50. Available from: http://www.liebertpub.com/doi/https://doi.org/10.1089/trgh.2016. from: www.ourcommons.ca. [Cited 27 Jan 2021] Available from: https://ontario.cmha.ca/documents/lesbian-gaybisexual-trans-queer-identified-people-and-mental-health/. [Cited 2021]. Available from: https://jamanetwork.com/journals/jamanetwor kopen/fullarticle/2719569. [Cited 5 Feb 2021] Available from: https://doi.org/10.15694/mep.2018.00000 